\title{
Transfert des contaminants dans le sous-sol saturé : principaux mécanismes en jeu et modélisation mathématique ou numérique adaptée
}

\author{
Contaminant transfer in aquifer systems : \\ operating mechanisms and applied mathematical or numerical modelling
}

par D. Pennequin

Safege Ingénieurs Conseils

Contaminant plume migration in aquifer systems can sometimes occur leaving no time for the pollutants to react with the transport medium, but more often these undergo a series of mechanical, physico-chemical or biological processes which lead to (1) plume spreading in both space and time, (2) plume degradation or even (3) transformation into other species which could be more or less toxic.

The relative weight of these mechanisms varies from one situation to the other and it is important to assess in each case the major operating processes in order to implement useful mathematical or numerical expressions for transport modeling.

Theoretical backgrounds are given and applications are made to two case studies.

\section{PRÉAMBULE}

La montée en puissance de l'industrialisation depuis la fin du siècle dernier a engendré une croissance notable des déversements de produits toxiques inhabituels dans le milieu naturel, conduisant souvent à sa contamination. Depuis une vingtaine d'années, une prise de conscience des dangers que ces comportements engendraient a permis de réduire fortement ces déversements dans les pays industrialisés, sans toutefois encore pouvoir les éliminer totalement. Aujourd'hui, l'héritage est constitué de milliers de sites pollués actifs ou abandonnés (friches industrielles), dont les contaminants débordent les limites pour s'infiltrer dans l'environnement et très souvent dégrader les nappes phréatiques, mettant en péril l'approvisionnement en eau potable du secteur.

L'alerte d'une pollution est le plus souvent donnée lors d'analyses réglementaires de routine effectuées sur les ouvrages d'eau destinée à la consommation humaine. Pour pouvoir réagir efficacement face à cette pollution et préserver la ressource en eau, une bonne connaissance de la nature des contaminants, de leur provenance, de leur mode et de leur vitesse de propagation s'avère être un atout précieux. Cela nécessite le plus souvent de recourir à une approche intégrée du problème, faisant notamment appel à un suivi analytique proprement dimensionné et à une modélisation mathématique du transfert des contaminants dans le sous-sol.
Le transfert des contaminants dans le sous-sol est conditionné par une série de mécanismes plus ou moins complexes, qu'il convient d'approcher par un schéma approprié fonction du contexte et des objectifs à atteindre.

Ces schémas peuvent donc varier d'une situation à une autre et les modélisations mathématiques doivent être adaptées en conséquence.

Compte tenu de la complexité du problème, il est proposé de se limiter ici à une discussion générale des principaux mécanismes de transfert ou affectant le transfert des polluants dans les milieux aquifères les plus courants, à une analyse de quelques techniques de modélisation de ces transferts avec leurs avantages et leurs limites d'utilisation, et à une présentation de deux cas de contamination souterraine et des techniques de modélisation utilisées.

II — PRINCIPAUX MÉCANISMES DE TRANSFERT OU AFFECTANT LE TRANSFERT DES POLLUANTS EN MILIEU SATURÉ

Le transfert des contaminants dans les nappes phréatiques s'effectue au gré des écoulements souterrains et des différents phénomènes connexes qui peuvent venir se greffer en 
cours de migration. Ces derniers sont principalement fonction de la nature des polluants et de la matrice de transfert, des conditions physico-chimiques du milieu et, pour les produits organiques, de la situation microbiologique du système.

\subsection{L'advection}

Le moteur de base du transfert des polluants en solution dans les aquifères est celui qui régit le déplacement de l'ensemble de la phase liquide ; c'est le champ de gravité. Ce mécanisme est appelé «advection » ou parfois « convection ". Le transport advectif des polluants peut être décrit en termes mathématiques comme suit :

$$
\text { Div } \mathrm{VC}+\eta^{\prime} \delta \mathrm{C} / \delta \mathrm{t}=0
$$

Où :

$\mathrm{V}=$ vitesse de Darcy des écoulements souterrains calculée à

l'aide de l'équation de diffusivité :

div $-\mathrm{k} \operatorname{grad} \mathrm{h}=\mathrm{S} \delta \mathrm{h} / \mathrm{dt}+\mathrm{R}$ avec :

$\mathrm{k}=$ perméabilité, $\mathrm{h}=$ charge hydraulique,

$\mathrm{S}=$ coefficient d'emmagasinement,

$\mathrm{R}=$ terme décrivant l'ensemble des échanges de flux avec l'extérieur du système

$\mathrm{C}=$ concentration du contaminant dissout dans la phase liquide

$\eta^{\prime}=$ porosité utile du milieu.

La vitesse effective du transport advectif des polluants dans les nappes correspond à la vélocité moyenne linéaire d'un front d'écoulement dans l'espace intersticiel connecté de la matrice poreuse ; c'est la vitesse de Darcy divisée par la porosité efficace du milieu aquifère $-\mathrm{V} / \eta$ ' [1].

Le transfert advectif des polluants s'effectue donc dans l'axe et à la vitesse des écoulements souterrains ; c'est très souvent le mécanisme moteur sinon dominant du transport de masse en milieu aquifère, et en particulier dans les nappes caractérisées par des vitesses d'écoulement rapides dues à de fortes perméabilités, avec des axes d'écoulement souvent variables dans le temps, et des temps de réaction avec la matrice d'accueil ou le milieu transporteur relativement lents.

\section{- 2.2 La dispersion hydrodynamique}

Un autre mécanisme vient souvent se greffer sur le transport advectif des contaminants et peut prendre de l'importance dans certains cas, notamment dans les milieux aquifères à moyenne et forte résistance avec des gradients hydrauliques importants, ou dans le cas de transferts peu mouvementés sur de longues distances; il s'agit de la dispersion hydrodynamique. Ce mécanisme est constitué de la conjugaison de deux phénomènes cinématiques : la dispersion mécanique et la diffusion moléculaire.

La dispersion mécanique est le résultat d'un processus de mélange mécanique turbulent lié notamment au contournement des grains et des hétérogénéités de la matrice aquifère par le polluant, lors de sa progression souterraine vers l'aval hydraulique. Ce processus est fonction de la géométrie intergranulaire et de l'organisation intra- et inter-faciès des différents corps géologiques traversés. Il dépend fortement de l'hétérogénéité du champ de vitesse dans l'aquifère et de ses fluctuations dans le temps.

La diffusion moléculaire ou ionique résulte d'un mouvement de type brownien des composés en solution. C'est un phénomène induit par des mouvements aléatoires cinétiquement entraînés par les gradients de concentration. Peu efficace dans la plupart des milieux aquifères, elle peut être le plus souvent négligée. Elle peut cependant prendre une relative importance dans les systèmes peu perméables (cas des stockages souterrains de produits dangereux).
Le processus de dispersion hydrodynamique des polluants contribue ainsi à leur étalement dans le temps et dans l'espace tri-dimensionel de l'aquifère. Le mécanisme opérationnel du transfert hydrodispersif (advection + dispersion) dans les milieux poreux ou simili-poreux est régi par l'équation ci-dessous :

$$
\text { Div D } \rho(\operatorname{grad} C / \rho)-\operatorname{div} \mathrm{V}^{\prime} \mathrm{C}=\rho \mathrm{C} / \rho \mathrm{t}
$$

Où :

$\mathrm{D}=$ tenseur de dispersion hydrodynamique (avec ou sans le terme de diffusion moléculaire)

$\rho=$ masse volumique du fluide

$\mathrm{C}=$ concentration en corps dissous

$\mathrm{V}^{\prime}=$ vitesse moyenne linéaire des écoulements souterrains $\left(\mathrm{V} / \eta^{\prime}\right)$.

La propriété dispersive du milieu est caractérisée par ses dispersivités longitudinale $\left(\alpha_{L}\right)$, transversale $\left(\alpha_{T}\right)$ et verticale $\left(\alpha_{\mathrm{v}}\right)$. Ces grandeurs qui peuvent théoriquement être déterminées sur le terrain, sont reliées au tenseur de dispersion par l'expression suivante :

$$
\mathrm{D}=\alpha\left|\mathrm{V}^{\prime}\right|+\mathrm{Df}
$$

Où :

$\left|V^{\prime}\right|=$ matrice des vecteurs d'écoulement (vitesse moyenne linéaire), par exemple, dans le système de coordonnées cartésiennes

Df $=$ coefficient de diffusion moléculaire (terme souvent négligé en milieu aquifère).

Il convient de souligner qu'en réalité la mesure de la dispersivité du milieu par des traçages sur le terrain reste un problème délicat sujet à de nombreuses controverses, notamment liées aux facteurs d'échelle et de parcours $[2,3,5]$.

\subsection{Les réactions avec le milieu}

Le transfert de contaminants en milieu aquifère peut également être affecté par des réactions de type physico-chimique ou microbiologique avec la matrice d'accueil, voire avec le milieu transporteur lui-même. Celles-ci peuvent être nombreuses et multiformes ; les principales cependant qui opèrent dans les milieux aquifères sont le plus souvent liées aux processus d'adsorption/désorption et de synthèse microbiologique. La discussion ici ne se bornera qu'à ces deux phénomènes.

\subsection{Adsorption/désorption}

L'érosion géochimique des corps géologiques conduit souvent à enduire les surfaces aux interfaces air-solide ou eausolide d'une pellicule de matière colloödale instable, amorphe ou sub-cristalline. Le milieu aquifère n'a pas échappé à cette règle.

Cette fine couche joue un rôle moteur dans les phénomènes d'échange ionique et plus particulièrement d'adsorption/désorption, à cause d'un ratio élevé entre les charges électriques et les surfaces des particules. Ce déséquilibre électrique est par ailleurs compensé par des ions de charge opposée, qui constituent la couche échangeable dans les processus d'adsorption/désorption. D'autres mécanismes entrent également en jeu et notamment pour le partitionnement des corps organiques neutres entre les phases liquide et solide (voir ci-après).

Ainsi lors de leur migration à travers les pores de la matrice aquifère, certains polluants peuvent temporairement être retenus par la phase solide sur les surfaces d'adsorption, avant d'être plus tard désorbés (réversibilité du processus) et retourner en solution dans la phase liquide. Compte tenu des relatives lenteurs de migration des contaminants dans les 
aquifères, le processus bi-directionnel d'adsorption/désorption a rapidement atteint son équilibre.

En admettant que l'équilibre soit atteint localement pour chaque point de l'aquifère, la connaissance de la répartition d'un polluant entre la phase solide (fraction adsorbée) et la phase liquide (fraction dissoute) permet de quantifier les réactions opérantes, et de prévoir le retard et les effets imputables aux mécanismes d'adsorption/désorption (diminution en aval des concentrations du polluant dans la phase liquide et étalement de son transfert dans le temps et dans l'axe d'écoulement).

Dans les cas simples, si le partitionnement d'un contaminant entre les phases liquide et solide peut se décrire au moyen d'un isotherme linéaire, la représentation mathématique généralement admise pour modéliser les réactions d'adsorption/désorption peut prendre la forme suivante :

$$
\Delta \mathrm{S} / \delta \mathrm{t} \gamma(1-\eta) / \eta=\mathrm{KaC}-\mathrm{Kb} \mathrm{S} \gamma(1-\eta) / \eta
$$

$$
\text { Où : }
$$

$\mathrm{S}$ : concentration dans la phase solide,

$\mathrm{C}$ : concentration dans la phase liquide,

$\gamma$ : poids spécifique de la matrice poreuse,

$\eta$ : porosité de la matrice aquifère,

$\mathrm{Ka}$ : constante décrivant la vitesse des réactions d'adsorption,

$\mathrm{Kb}$ : constante décrivant la vitesse des réactions de désorption.

Dans les cas plus compliqués des isothermes non-linéaires de partition (ex. Langmuir, Freundlich,...), les constantes Ka et $\mathrm{Kb}$ peuvent devenir des fonctions plus ou moins complexes.

Il apparaît cependant que les isothermes de partitionnement linéaires ou sub-linéaires (facilement linéarisables) sont applicables pour une partie importante des micropolluants organiques communs, dont la plupart des composés organiques hydrophobes neutres et des polluants organiques dits "prioritaires" de l'EPA. Dans ces cas d'ailleurs, le mécanisme d'adsorption/désorption reste essentiellement un processus de partage entre la phase liquide et la matière organique contenue à la périphérie du substrat solide. La capacité d'adsorption (coefficient de partitionnement) d'une de ces substances peut être approchée et décrite alors plus spécifiquement sur la base de son coefficient de partage octanol-eau souvent référencé dans la littérature, et du contenu en carbone organique de la matrice aquifère. Son expression mathématique est alors du type :

$$
\log \mathrm{Kp}=\mathrm{a} \log \mathrm{Koe}+\log \mathrm{Fco}+\mathrm{b}
$$

$$
\text { Où : }
$$

$\mathrm{Kp}=$ coefficient de partitionnement liquide-solide à l'équilibre de la substance considérée,

Koe $=$ coefficient de partitionnement à l'équilibre de la substance considérée entre le n-octanol et l'eau,

$\mathrm{Fco}=$ fraction de carbone organique contenu dans le substrat solide,

$a, b=$ coefficients empiriques.

Il semblerait qu'en cas de teneur très faible en carbone organique dans la fraction fine du substrat solide, les mécanismes d'adsorption/désorption soient plutôt orientés par des interactions entre la phase liquide et la fraction argileuse du substrat solide.

Le problème d'adsorption des métaux toxiques est généralement beaucoup plus complexe ; ces derniers dans leurs formes les plus courantes sont toutefois souvent moins mobiles dans les nappes phréatiques.

Enfin, il convient d'attirer l'attention sur le fait que ces schématisations, souvent nées d'expériences menées en laboratoire, ont forcément leurs limites d'utilisation en milieu aquifère [2]; il n'en demeure par moins qu'appliquée avec discernement, et croisée avec d'autres informations, elles donnent souvent de bons résultats.

\subsubsection{Synthèses microbiologiques}

Les réactions de synthèse microbiologique qui peuvent prendre place dans les milieux aquifères sont multiples et multiformes.

Dans le cas des polluants, elles peuvent conduire à leur dégradation ou encore à leur transformation en d'autres substances inertes ou toxiques. Parmi ces nombreuses réactions potentielles, on peut noter par exemple l'hydroxylation des composés aromatiques, comme le benzène, dans le cadre de réactions oxydantes, ou encore la déshydrochloration du DDT qui entraîne la formation de DDE pour les réactions réductrices.

Le déclenchement, les vitesses et l'arrêt des réactions de synthèse microbiologique dépendent d'un grand nombre de facteurs, dont la concentration du ou des polluant(s), l'environnement physico-chimique du système, la gamme de microorganismes pré-existants, leur potentiel et temps d'adaptation, leur seuil d'activation, les niveaux de concentration létale, le contexte de rivalité entre microorganismes,...

Sans entrer dans plus de détails, ces quelques considérations d'ordre général montrent déjà la complexité du sujet. Alors que beaucoup d'effets unitaires semblent aujourd hui à peu près compris et prévisibles dans le cadre d'un contexte maîtrisé (en laboratoire), la superposition de l'ensemble des mécanismes qui opèrent dans le milieu aquifère, dont les paramètres sont souvent mal connus, rend délicates et très difficiles leur estimation et leur prévision, et par là même leur représentation mathématique.

Les modèles du type Monod ou équivalent développés en laboratoire peuvent donc difficilement être appliqués au milieu aquifère sans courir le risque de tomber dans une représentation illusoire.

\section{III — MODÉLISATION MATHÉMATIQUE APPLIQUÉE AU TRANSFERT DES POLLUANTS}

La modélisation du transfert des contaminants en milieu poreux ou simili-poreux doit pouvoir répondre aux objectifs fixés dans chaque cas de figure, et représenter correctement le fonctionnement du système concerné, pour pouvoir apporter les réponses et solutions attendues. A ce titre, elle doit pouvoir prendre en compte, au moins d'une manière ou d'une autre les principaux mécanismes opérants et/ou leurs effets.

\section{- 3.1 Représentation mathématique des principaux mécanismes de transfert des polluants et expres- sion mathématique de base du modèle courant}

Les représentations mathématiques du transfert des polluants prises en compte dans les modèles peuvent inclure différents niveaux de difficultés en fonction du système simulé et du degré de complexité retenu pour l'expression des mécanismes en jeu. Dans le cas présent, seule la formulation la plus couramment utilisée est abordée.

L'expression mathématique des différents mécanismes qui régissent le transfert des contaminants dans les nappes doit avant tout rester fidèle à leur fonctionnement et/ou à leur conséquences. Dans le cas de l'advection et de l'hydrodispersion, les équations mathématiques données précédemment (éq. 1 et 2) peuvent être directement adaptées et utilisées dans le modèle. Ce dernier pourra alors simuler selon le cas le fonctionnement du transfert advectif ou hydrodispersif des polluants dans l'aquifere. 
L'adsorption/désorption par contre est généralement prise en compte dans les modèles par le biais d'un facteur de retard; on représente alors ici la conséquence du phénomène et non les mécanismes de fonctionnement (rappelons que les phénomènes d'adsorption/désorption ont pour effet de retarder la migration des polluants). L'expression mathématique la plus utilisée est :

$$
\mathrm{Rd}=\mathrm{V}^{\prime} / \mathrm{Vr}=1+\mathrm{Kp}\left(\gamma / \eta^{\prime}\right)
$$

Où :

$\mathrm{Rd}=$ facteur de retard

$\mathrm{Vr}=$ vitesse de migration réelle des contaminants adsorbables (avec $\mathrm{Vr}<\mathrm{V}^{\prime}$ ).

Le facteur de retard peut parfois directement être déterminé au moyen d'historiques de données collectées sur le terrain (ex. analyse de l'évolution différentielle des concentrations entre espèces mobiles et adsorbables par rapport à un temps d'introduction des contaminants dans la nappe, au champ des vecteurs de flux,...) [9] ou plus souvent, indirectement dans le cadre d'une expérimentation effectuée en laboratoire (ex. obtention de $\mathrm{Kp}$ à partir d'une colonne de percolation),

Cette technique a l'avantage d'être relativement simple à mettre en œuvre dans une modélisation [9] ; elle le devient moins à partir du moment où l'isotherme d'adsorption du polluant s'écarte significativement du schéma linéaire.

La biodégradation de la matière polluante ou sa transformation en matière neutre sont les phénomènes de synthèse microbiologique qui nécessitent le plus souvent d'être pris en compte dans les modèles mathématiques. En général, ils sont représentés sous la forme d'une fonction de décroissance linéaire du type :

$$
\delta \mathrm{C} / \delta \mathrm{t}=\lambda \mathrm{C}
$$

ou parfois :

$$
\lambda=\ln (2) / \mathrm{Th} \text { avec } \mathrm{Th}=\text { la demi-vie de l'espèce considérée. }
$$

Des valeurs de demi-vie de plusieurs espèces polluantes peuvent être trouvées dans la littérature; cependant une grande prudence doit être exercée lors de leur utilisation, car ces valeurs ont pour la plupart été obtenues dans le cadre d'expérimentations effectuées en laboratoire, dans des milieux maîtrisés, avec des substrats de composition simple. Cette approche très utilisée court le risque de s'avérer trop simpliste dans beaucoup de cas; elle a le mérite néanmoins de pouvoir considérer le phénomène au moins de manière approximative. Lorsque cela s'avère possible, il est préférable de la croiser avec des données obtenues sur le terrain (historique spatio-temporel des concentrations, ....).

Il en résulte que l'expression mathématique de base, utilisée le plus souvent pour représenter la modélisation mathématique du transfert des contaminants sous l'influence des quatre phénomènes décrits ci-dessus, peut s'écrire de la manière suivante :

$$
\begin{aligned}
& \operatorname{Div} D \rho(\operatorname{grad} C / \rho)-\operatorname{div} V^{\prime} C= \\
& \operatorname{Rd} \delta C / \delta t+\lambda C \operatorname{Rd}-C^{\prime} W^{*} / \eta^{\prime}
\end{aligned}
$$

où :

$C^{\prime}=$ concentration de la source de pollution ou concentration extraite du système considéré,

$\mathrm{W}^{*}=$ terme représentant les échanges de flux et de masse avec l'extérieur (ex. flux de pollution introduit dans la nappe).

La représentation mathématique donnée dans l'équation (8) inclut l'ensemble des phénomènes décrits ci-dessus ; dans de nombreux cas, les objectifs à atteindre ou la prépondérance d'un seul ou de deux mécanismes de transfert ne nécessitent pas la prise en compte de tous les aspects du transport. L'expression mathématique de base du modèle peut alors être simplifiée en conséquence.

\subsection{Principaux types de modèles}

Il existe en général deux grandes familles de modèles utilisés pour simuler les problèmes courants de pollution dans les nappes ; les modèles dits " mathématiques " ou " analytiques ", et les modèles dits "numériques".

Dans la première famille de modèles, la ou les équations mathématiques sont résolues par des méthodes analytiques. $\mathrm{Ce}$ sont donc des modèles en principe exacts. Cependant la résolution mathématique de la ou des équations du modèle, nécessite un grand nombre d'hypothèses simplificatrices. En règle générale, les modèles analytiques ne sont applicables que pour des systèmes aquifères très simples (homogènes, isotropes, géométrie parfaitement rectangulaire, source de pollution instantanée....) et d'extension réduite. Ils peuvent parfois aussi être utiles dans le cadre d'une approche préliminaire d'un problème de contamination pour lequel des réponses urgentes sont demandées.

La deuxième catégorie de modèles permet de prendre en considération des cas de figures beaucoup plus complexes, incluant hétérogénéités, anisotropies et géométries irrégulières comme c'est souvent le cas dans les milieux aquifêres. Les systèmes d'équations nécessaires pour représenter correctement l'aquifere et son fonctionnement ne peuvent alors pas être résolus de manière globale par une méthode analytique.

La résolution des équations cette fois s'effectue par une méthode numérique dans un espace discrétisé ; un maillage est effectivement appliqué sur le secteur modélisé. C'est à travers ce dernier que les variations des propriétés de l'aquifère peuvent être prises en compte ; les paramètres maintenus obligatoirement constants à l'intérieur d'une même maille, par exemple, peuvent varier d'une maille à l'autre.

Le schéma numérique le plus utilisé est celui des différences finies, les éléments finis étant souvent plus compliqués à mettre en œuvre pour un rendu semblable. Les méthodes de résolution sont le plus souvent du type itératif ou semi-itératif, voire parfois direct.

Les modèles numériques sont moins précis que les modèles analytiques sur un plan strictement mathématique (ce sont effectivement des solutions approchées), mais ils le sont nettement plus en réalité et sur le plan pratique, car ils permettent la prise en compte d'une représentation beaucoup plus fiable du système aquifère considéré.

La simulation numérique de l'évolution des contaminants dans les nappes nécessite au préalable d'établir la configuration hydraulique du système aquifère ; un modèle numérique de transport de masse est donc constitué d'un modèle hydrodynamique de base, sur lequel se greffent ensuite les différents modules secondaires représentant selon les besoins, l'hydrodispersion et les mécanismes de réaction avec les phases liquides et solides du support aquifère.

\subsection{Quelques pièges potentiels de la modélisation}

Un modèle ne vaut que par la qualité des données à partir desquelles il a été conçu. Une grande attention doit donc être portée aux données. Dans chaque cas les données nécessaires restent fonction des caractéristiques du contexte, des objectifs à atteindre et de la précision recherchée. Trop peu de données peuvent souvent amener à s'appuyer sur des hypothèses trop vite oubliées, et générer des schémas de calages numériques ou mathématiques multiples.

L'établissement des limites dans le cas des modèles numériques restent par ailleurs une opération délicate ; ces dernières doivent être placées de manière à minimiser les effets dits "de bord ", qui peuvent entraîner des erreurs importantes lorsque dans les simulations prospectives, par exemple les contextes d'entrées - sorties du système, sont 
amenés à différer fortement du contexte de base utilisé pour l'ajustement du modèle.

Un modèle numérique, comme d'ailleurs un modèle analytique, doit pouvoir simuler fidèlement le fonctionnement du système aquifère qu'il représente. A cet effet, avant de servir, il doit autant que possible être étalonné sur la base d'un ensemble minimum de points de contrôle, fonction du problème à traiter. Cet aspect reste trop souvent négligé et conduit parfois à des conclusions erronées avec le cortège de conséquences qui en découlent, et notamment des investissements inutiles.

Dans certains cas, les algorithmes de résolution des équations peuvent converger vers des solutions erronées, transparentes à l'utilisateur néophite.

\section{EXEMPLES DE MODÉLISATIONS UTILISÉES DANS DEUX CAS DE POLLUTION}

Le choix du type de modèle adapté à un cas de figure donné est fonction d'un certain nombre d'éléments, et notamment des problèmes à résoudre, des caractéristiques du système aquifère, des objectifs poursuivis et de la précision attendue. Deux exemples sont donnés ci-dessous pour illustrer ce propos.

\section{- 4.1 Pollution d'une nappe de sable dans le sud-ouest de la France}

\subsubsection{Situation du problème}

Un incident dans un petit entrepôt de produits toxiques, situé quelques centaines de mètres en amont d'un secteur maraîcher doté de quatre puits, a conduit à introduire plusieurs $\mathrm{m}^{3}$ d'une solution de chlorobenzene (composé organique hydrophobe neutre) dans une nappe de sable du sud-ouest de la France.

Très inquiètes, les autorités locales souhaitaient savoir si le polluant pouvait atteindre la zone de maraîchage, et en particulier contaminer les quelques puits existants, et dans l'affirmative au bout de combien de temps et pendant combien de temps.

\subsubsection{Les objectifs}

Les objectifs consistaient à évaluer le risque de contamination des puits maraîchers, et le cas échéant estimer de manière approximative le degré, la période et la durée de contamination de chaque ouvrage.

\subsubsection{Les données disponibles}

La nappe de géométrie peu chahutée, épaisse de quelques mètres seulement et recouverte d'une fine couche de limon était relativement homogène entre l'entrepôt et la zone de maraîchage. L'axe d'écoulement a été déterminé (sud-ouest) et la vitesse régionale d'écoulement a été évaluée entre environ 8 et $12 \mathrm{~m} / \mathrm{j}$ à partir de données collectées sur le terrain. La valeur des autres paramètres a fait l'objet d'estimations à partir de la littérature, de bases de données et de l'expérience obtenue dans des contextes semblables. Notons ainsi en particulier que la porosité a été fixée à $8 \%$, la dispersivité longitudinale $\left(\alpha_{L}\right)$ à $50 \mathrm{~m}$ (relation de première approche de Gelhar et al. [3] - modifiée), le ratio $\alpha_{\mathrm{L}} / \alpha_{\mathrm{T}}$ à 3 et les coefficients « $a$ » et « $b$ » de l'équation (5) pour déterminer $\mathrm{Kp}$, à 0,72 et 0,49 respectivement conformément aux résultats obtenus par Schwartzenbach et al. [7]. La biodégra- dation a été négligée pour deux raisons; le chlorobenzene semble souvent relativement peu biodégradable dans le milieu souterrain et surtout une approche conservatrice du problème était souhaitée. La volatilisation du polluant (mécanisme non abordé ci-dessus) n'a pas été prise en compte du fait de la présence d'une couche de limons superficiels et dans la même optique d'approche conservatrice.

\subsubsection{Choix du type de modèle}

Les réponses étaient à fournir rapidement dans le cadre d'une expertise préliminaire. La précision pouvait donc être approximative.

Compte tenu de l'ensemble du contexte décrit ci-dessus, le choix s'est donc porté sur un modèle analytique. Le modèle retenu a été celui de Wilson et Miller (1978) développé notamment pour un système aquifère rectangulaire, homogène, à écoulement stabilisé en $\mathrm{x}$, dans lequel le contaminant est introduit de manière instantanée sur toute la hauteur saturée. Ce modèle fait également l'hypothèse que l'isotherme d'adsorption et la dégradation du contaminant suivent un schéma linéaire. Son expression mathématique est résumée ci-dessous :

$$
\begin{aligned}
& C(x, y, t)=\left(\operatorname{Co} Q^{\prime} / 4 \pi \eta b t \sqrt{D x D y}\right) \\
& \exp \left( \pm \lambda t \pm\left(x R d \pm V^{\prime} x t\right)^{2} / 4 D x t R d \pm\left((y R d)^{2} / 4 D y t R d\right)\right)
\end{aligned}
$$

où :

Co : concentration initiale du contaminant à son introduction dans la nappe

$\mathrm{Q}^{\prime}$ : volume de contaminant introduit dans la nappe,

b : épaisseur saturée,

$\mathrm{t}$ : le temps écoulé depuis l'introduction du polluant dans la nappe,

$\mathrm{x}, \mathrm{y}$ : coordonnées spatiales,

Dx,Dy : coefficients de dispersion en $x$ et en $y$,

$V^{\prime} x$ : vitesse moyenne linéaire des écoulements souterrains en $x$.

\subsubsection{Résultats obtenus}

Les résultats obtenus ont montré que le chlorobenzene commencerait à atteindre le premier puits $(\mathrm{P} 1) 11$ jours après l'incident, que les trois puits en amont (P1, P2 et P3) resteraient contaminés avec des teneurs supérieures à $1000 \mathrm{mg} / \mathrm{l}$ pendant une durée d'environ 230 à 300 jours, avec des concentrations maximum pouvant atteindre 2000 à $6000 \mathrm{mg} / \mathrm{l}$ (fig. l). A titre d'indication, la recommandation

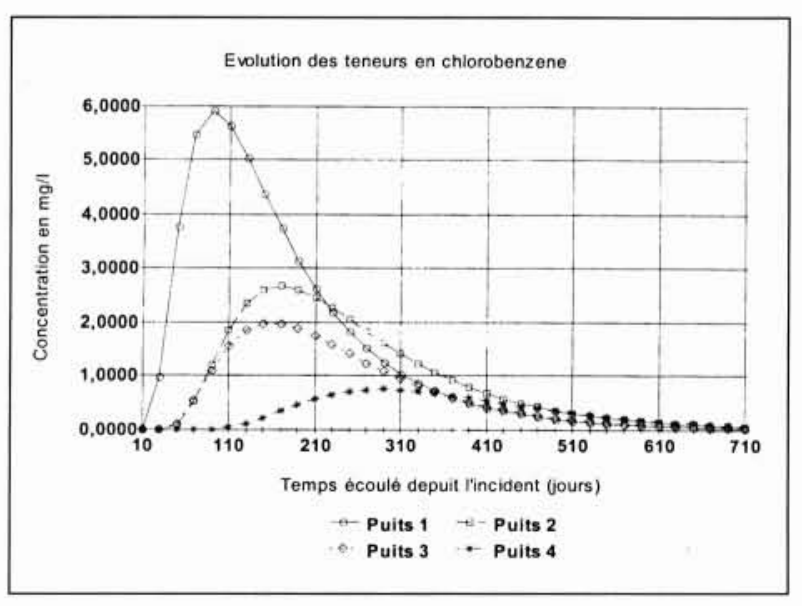

\section{Puits maraîchers}


OMS pour le chlorobenzene est fixé à $300 \mathrm{mg} / \mathrm{l}$ pour l'eau potable.

Face à cette perspective une solution de confinement hydraulique et de dépollution a été mise en place en aval du dépôt.

\subsection{Pollution de la nappe du Rhône}

\subsubsection{Situation du problème}

Le deuxième cas de figure est très différent de par sa taille, son contexte et sa dynamique. Un complexe de l'industrie chimique avait fortement pollué la nappe alluviale du Rhône en rive gauche, notamment en relargant dans l'environnement par le biais de lagunes d'infiltration, des produits phytosanitaires entrant dans la fabrication de pesticides. Cette nappe était exploitée au moyen d'une cinquantaine de puits et de forages à grands débits, à la fois pour l'alimentation en eau potable et la fourniture d'eau industrielle.

Depuis l'apparition de la pollution en 1993, un dispositif de protection des captages d'eau potable avait été mis en place et des mesures de réduction du débit d'exploitation avaient été prises ; ainsi dans le principal champ captant fournisseur d'eau potable, le débit de production était passé de 30000 à 15000 , voire $10000 \mathrm{~m}^{3} / \mathrm{j}$. Cette situation et un accroissement des besoins en eau potable n'allaient pas sans créer des tensions en période estivale, et le spectre d'un manque d'eau a donné naissance à un projet de nouveau champ captant à quelques centaines de mètres en aval sur la rive droite du fleuve.

Ce projet suscitait néanmoins quelques inquiétudes auprès des administrations chargées d'instruire et/ou de statuer sur le dossier de demande d'autorisation d'exploitation et de mise en place des périmètres de protection réglementaire ; en effet, la crainte avait été soulevée d'un risque d'entraînement des polluants de la rive gauche vers la rive droite du Rhône, sous l'effet des nouveaux pompages.

\subsubsection{Objectifs}

Les objectifs dans ce deuxième exemple étaient triples : établir la possibilité ou non d'un transfert souterrain sous le fleuve, et dans l'affirmative, évaluer les flux hydrauliques et massiques qui pourraient être induits par les ouvrages prévus, et définir les moyens de protection à mettre en place. Le travail devait s'effectuer dans le cadre du risque maximum.

\subsubsection{Données disponibles}

Les données disponibles étaient nombreuses et incluaient notamment des historiques piézométriques et d'analyse de la pollution, les historiques des principaux débits exploités sur le secteur, des résultats de pompages d'essais et les résultats de plusieurs investigations géoélectriques et sondages mécaniques réalisés sur l'ensemble de la zone d'intérêt.

L'analyse de ces données a permis de mettre en évidence à la fois la macro-homogénéité relative du système aquifère de part et d'autre du fleuve, et ses nombreuses meso- et micro-hétérogénéités tri-dimensionnelles liées notamment à la présence de plusieurs paléochenaux, parfois entrecroisés, à d'anciennes terrasses alluvionnaires et à la morphologie très accidentée du substratum. L'épaisseur de la nappe variait ainsi par exemple de 2 à plus de $35 \mathrm{~m}$ et sa transmissivité de $10^{-1}$ à $10^{-4} \mathrm{~m}^{2} / \mathrm{s}$.

Une double prospection bathymétrique et acoustique a en outre permis de mettre en évidence la prolongation de l'aquifère sous le Rhône par le biais d'une fine tranche d'alluvions grossières (environ 1 à $3 \mathrm{~m}$ d'épaisseur). Enfin, un premier modèle de gestion de la pollution en rive gauche avait démontré la rapidité des écoulements souterrains et surtout, leur réorientation fréquente face aux déplacements incessants des centres de gravité de pompage en nappe [4]. Il avait également démontré qu'une partie importante de l'eau pompée provenait soit du fleuve, soit de la rive opposée, soit des deux à la fois.

\subsubsection{Choix du type de modèle}

Les caractéristiques complexes du système aquifère et la difficulté des questions à résoudre ont nécessité la construction d'un modèle numérique en 3 dimensions, dans lequel notamment les entités hydrologiques ont été clairement séparées des horizons hydrogéologiques sur le plan numérique, tout en restant connectées à ces derniers au niveau hydraulique au moyen d'une limite de type Fourier. Les nombreuses hétérogénéités ont été représentées par des mailles de taille variable, dans chacune desquelles les valeurs correspondantes des paramètres ont été introduites.

La réorientation constante des écoulements souterrains diminue fortement le poids des phénomènes de dispersion hydrodynamique et maximise les transferts advectifs. En outre, le premier modèle avait permis non seulement de bien retracer l'évolution observée des contaminants dans la nappe, mais également de remonter aux sources de pollution et d'implanter un dispositif de protection efficace sur la base de la seule considération du transfert advectif [4]. L'hydrodispersion n'a donc pas été prise en compte dans ce nouveau modèle.

Enfin, les nombreux contaminants encore piégés en rive gauche du fleuve et le choix d'une approche prudente et donc maximaliste dans le cas présent (évaluation du flux massique maximum de polluants vers la rive droite), a conduit à négliger les phénomènes d'adsorption/désorption et de biodégradation.

L'expression mathématique de base du modèle décrite dans l'équation (8) s'est donc résumée à :

$$
-\operatorname{div} \mathrm{V}^{\prime} \mathrm{C}=\delta \mathrm{C} / \delta \mathrm{t}-\mathrm{C}^{\prime} \mathrm{W}^{*} / \eta^{\prime}
$$

\subsubsection{Résultats obtenus}

Le modèle a permis de montrer que des flux souterrains migreraient sous le Rhône sous l'effet des nouveaux pompages en rive droite, que ces flux représenteraient dans le cas le plus défavorable environ $5 \%$ de l'eau prélevée par les nouveaux forages, et que les teneurs en polluants, si tel était le cas, n'atteindraient pas les seuils de concentration maximum admissible fixés dans le cadre de la réglementation en vigueur.

Ces résultats ont été obtenus sur la base des concentrations maximum en polluants mesurées alors en rive gauche du fleuve. Des mesures de dépollution ayant été prises, il était raisonnable de penser que ces concentrations diminueraient dans le temps. Cependant les normes réglementaires pouvaient également se resserrer et des relargages accidentels ne pouvaient pas être exclus. Un dispositif de protection approprié des nouveaux captages a donc été élaboré à l'aide de simulations effectuées sur le modèle; celui-ci comporte des ouvrages de piégeage et/ou de surveillance de la pollution implantés de part et d'autre du fleuve (fig. 2). Il est à noter que ce dispositif très particulier est lié à la configuration tout aussi particulière des écoulements souterrains dans le secteur, et à un choix de politique de prudence de la part de l'ensemble des intervenants. 
2. Dispositif de protection contre I'entraînement d'une pollution chimique de la rive gauche du Rhône.

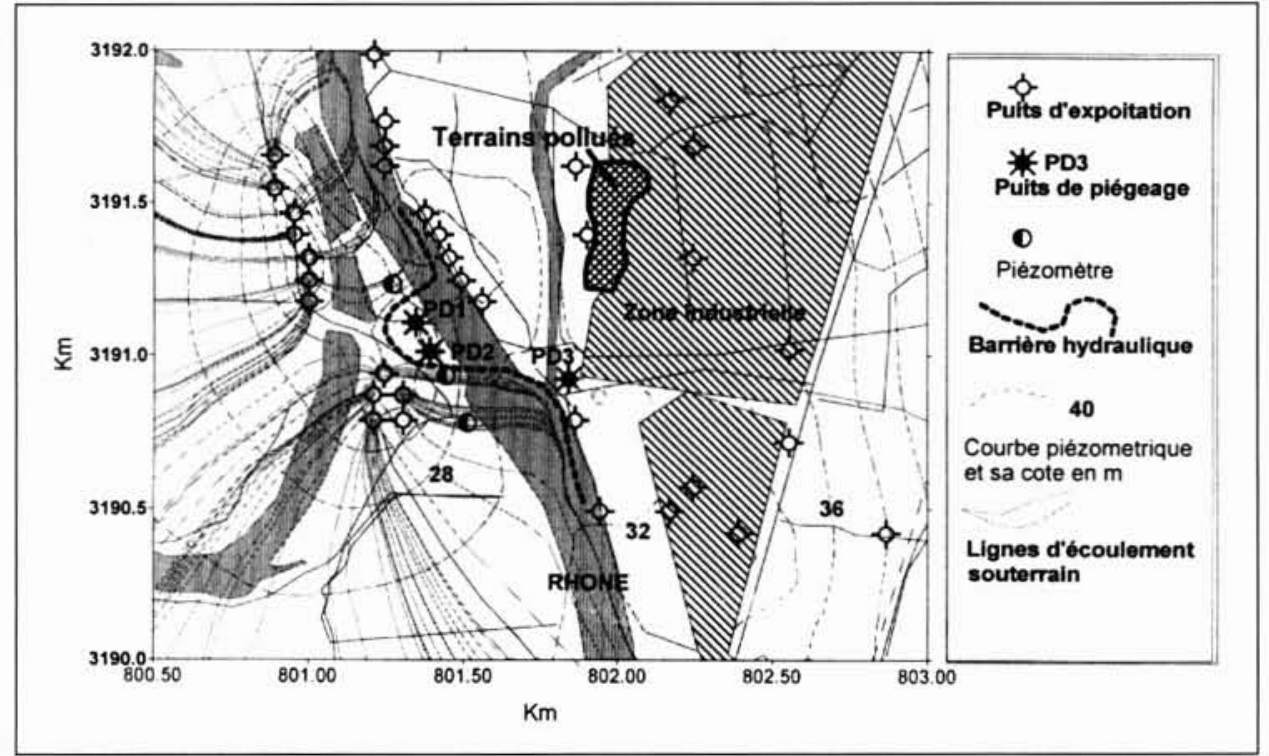

\section{$\mathrm{V} \square \mathrm{CONCLUSION}$}

Le transfert des contaminants dans les milieux aquifères reste avant tout étroitement lié aux écoulements souterrains. Dans de nombreux cas, sur ce mouvement de base viennent se greffer un certain nombre d'autres phénomènes ou mécanismes qui contribuent à les étaler dans l'espace, à ralentir leur progression ou encore à les dégrader, voire à les transformer en d'autres substances.

Bien que tous les phénomènes ou mécanismes de transfert ne soient pas encore vraiment maîtrisés, la prise en compte de l'advection, de la dispersion hydrodynamique, de l'adsorption/désorption et/ou de la dégradation souvent suffisent pour pouvoir utilement décrire et simuler leur progression dans les milieux aquifères, au moins de manière approximative. Cette schématisation dans le cadre de modélisations mathématiques ou numériques permet d'obtenir dans de nombreux cas des éléments essentiels de réponse aux questions posées, et notamment concernant le devenir des polluants, les risques de contamination des ressources en eau, l'identification des sources de pollutions et les dispositifs de protection ou de dépollution à mettre en place.

Les modèles mathématiques ou plus souvent numériques restent en effet les meilleurs outils pour traiter les problèmes de transfert de polluants dans les nappes souterraines, et en particulier parce qu'ils permettent d'intégrer l'ensemble des principaux aspects et mécanismes du fonctionnement des systèmes aquiferes, ce qui est nécessaire pour à la fois pouvoir assurer les approches d'ensemble et de détail qu'imposent la plupart des contextes de pollution. Les modèles sont en effet de puissants outils d'analyse, de synthèse, d'anticipation et d'aide à la décision.

Par contre, il est essentiel que les modèles utilisés pour la résolution des problèmes de contamination soient correctement structurés, dimensionnés, étalonnés et adaptés au contexte concerné, sous peine d'entraîner une cascade de mauvaises décisions, des investissements inutiles, voire la condamnation à terme d'une ressource en eau; ils ne doivent être ni erronés, ni trop schématiques, ni d'une complexité illusoire.

\section{RÉFÉRENCES}

[1] Alessandrello E, Pennequin D, De Meredieu J et GUYONNET D : « Modélisation appliquée au transfert des polluants dans le sous-sol ", Question 3, rapport $n^{\circ} 8$, Congrès $\mathrm{XXI}^{\mathrm{e}}$ Journée de l'Hydraulique-Sophia Antipolis 29-31 Janvier 1991

[2] Anderson M.P. et Woessner W.W. : «Applied Groundwater Modeling - Simulation of Flow and Advective Transport " , Academic Press Inc., San Diego Ca. 1992

[3. Gelhar, L.W., Mantoglou A., Welty C. et Rehfeldt K.R. : A review of Field Scale Subsurface Solute Transport Process Under Saturated and Unsaturated Conditions. Electric Power Research Institute, Palo Alto, Ca., 107 p., - 1985

[4] Pennequin D: «Flowlines and Plume Evolution Analysis for Well Field Protection ", World Water and Environmental Engineering - Janvier 1997

[5] Safege : Synthèse de l'Etat de l'Art sur la Connaissance des Différents Phénomènes Régissant la Migration d'Agents Pollueurs en Milieu Poreux ou Simili-Poreux Saturé " - rapport en cours d'élaboration.

[6] Whitaker S: «Diffusion and Dispersion in Porous Media ". Journal of American Institute of Chemical Engineering, vol. 13, $n^{\circ} 3$, p. 420-427 - 1967

[7] Swartzenbach R.P. et Westhall, J. Sorption of Hydrophobic Trace Organic Compounds in Ground Water Systems. Water Science Technology, vol. 17, p. 39-55 - 1985

[8] Wilson J.T et Miller P.J. : « Two Dimensional Plume in Uniform Ground-Water Flow ». Journal of Hydraulic Division, ASCE, vol. $104, n^{\circ} 4$, p. 503-514- 1978

[9] Pennequin D : «Combined field and mathematical approach to protect two drinking water production well fields from contaminant plume migration $»$. Annales du Congrès International "First World Water Congress of the International Water Association JWA-AGHTM-CERP", 3-7 juillet 2000, Paris, vol. 5 , p $417-426$ 